\title{
Process-annotated Service Discovery facilitated by an n-gram-based index
}

\author{
Bendick Mahleko $^{1} \quad$ Andreas Wombacher ${ }^{2 *}$ \\ Peter Fankhauser ${ }^{1}$ \\ ${ }^{1}$ Fraunhofer Institute, Integrated Publication and Information Systems (IPSI) \\ D-64293 Darmstadt, Germany, http://ipsi.fraunhofer.de/oasys/ \\ \{mahleko|fankhaus\}@ipsi.fraunhofer.de \\ ${ }^{2}$ Center for Telematics and Information Technology, University of Twente \\ 500 AE Enschede, The Netherlands, a.wombacher@utwente.nl
}

\begin{abstract}
Service discovery in web service infrastructure must consider abstract processes to guarantee successful business interactions with the matched services. Unfortunately, matchmaking process annotations requires a significant processing effort, thus, finding relevant service providers in a large collection is time consuming and requires more efficient access methods than sequential scanning. The paper presents an approach supporting more efficient querying of abstract processes by introducing an abstraction to reduce the computational complexity of the comparison operation. Experimental studies on a collection of over 800 abstract business processes derived from the RosettaNet PIP specification show that our approach outperforms naive sequential scanning by up to an order of magnitude.
\end{abstract}

\section{Introduction}

Web services and related technologies promise to facilitate the efficient execution of B2B e-commerce by integrating business applications across networks like the Internet. As web services are advertised as a technology for implementing loosely coupled business processes, that is a dynamic and flexible binding of services, the support of finding relevant service providers is a major success criteria. This service discovery aspect is based on meta-data annotated to services. While the semantic web community [2] adds semantic annotations describing the service functionality [14], approaches like [16] extend the UDDI data model by additional properties assigned to the service. Despite all

\footnotetext{
* This work has been done during employment at Fraunhofer IPSI.
}

this effort, the problem of finding services that guarantee a successful business interaction is not supported by these approaches.

The W3C web service architecture proposes to use abstract business processes as service annotation to decide whether services are guaranteeing a successful business interaction. In particular, abstract business processes are workflows comprising choreographed messages which cannot be handled by traditional database search approaches. A more complex search operator is required for querying than traditional attribute/ value searches. The above described problem concludes in the following questions:

- How to determine if two abstract business processes guarantee a successful business interaction?

- How to efficiently find compatible business processes from a large collection?

The first question is partially addressed in [19], where abstract business processes are represented as finite state automata (FSA) [8] and a non-empty intersection guarantees successful business interactions due to a common execution sequence of messages.

This paper focuses on the second question. It presents an indexing approach to enable an efficient calculation of compatible business processes from a large collection, where non-empty intersection is the matching criteria. So, the query consists of an abstract business process and the optimal result list contains all matching abstract business processes. Compared to a sequential scan of a collection the presented approach allows query evaluation on behalf of meta-data rather than the process models themselves - this is also known as indexing.

The approach is based on the assumption that the different providers are using a common semantics on message names. This assumption is reasonable because several standards exist (e.g. OTA, RosettaNet, IOTP etc.) where different organizations agreed on the semantics of a set of mes- 
sages. Thus semantic reasoning on messages is not required.

The rest of the paper is organized as follows: Section 2 illustrates matchmaking on behalf of a concrete example, Section 3 gives an overview of FSA and the definition of matchmaking, Section 4 discusses existing approaches, Section 5 presents our approach, and Section 6 describes the implementation and evaluation illustrating that our indexing approach outperforms sequential scanning by up to an order of magnitude. Section 7 concludes the paper.

\section{Example}

Figure 1 depicts two abstract business processes describing a procurement scenario. Nodes represent the states of an abstract business process; the end states are identified by a double circle. Edges represent state transitions, which are labeled with messages being correlated to RosettaNet Partner Interface Processes (PIPs) annotated in brackets to represent semantic equivalence by string equivalence. Figure

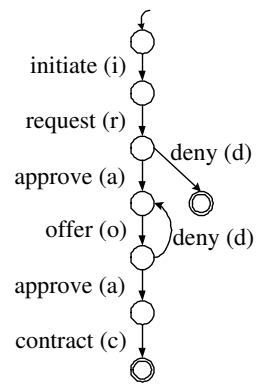

(a)

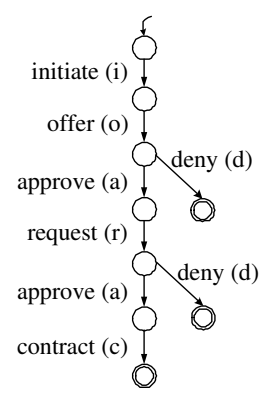

(b)

\section{Figure 1. (a) Customer Process. (b) Account- ing Process}

1(a) shows the customer business process, where the customer initiates the process by an initiate message (3A1), followed by a purchase request (3A4) sent to the accounting. The accounting either approves (approve message (3A7)) the request or denies (deny message (3A9)) it. In case of approval, the customer collects offers from merchants and presents the condensed offer to the accounting, which either accepts (approve message (3A7)) or rejects (deny message (3A9)) the offer. In case of acceptance, the customer is allowed to sign the contract by sending a contract message (3A6).

Opposed to the customer process, the accounting process insist on the knowledge of a concrete offer before, finally, deciding on the request itself. Thus, the sequencing of the order and request message is changed compared to the customer business process.
At the level of individual messages these two business processes match. However, because they require a different sequencing of offer and request messages, they are incompatible, that is, they can not successfully interact. In order to avoid matching incompatible business processes we thus need to take into account message sequences rather than individual messages.

\section{Efficient Business Process Matchmaking}

To be able to describe the above motivated problem more precisely, a formal model of abstract business processes is required.

\subsection{Formal Model}

Finite state automata (FSA)[8] constitute a suitable starting point to model business processes for the purpose of matchmaking. Where matchmaking is based on non-empty intersection of finite state automata. They can represent (possibly infinite) sets of message sequences without considering branching conditions and parallel execution capabilities as provided by more expressive approaches such as Petri Nets. While Petri Nets are also closed under intersection [15], they require a much higher computational complexity compared to FSA. In particular, Petri Nets allow concurrent execution resulting in non-polynomial computation times for reachability and liveness problems [4]. If the Petri Net class is limited to bounded ${ }^{1}$ nets, several polynomial results exist. In case of bounded nets the reachability graph can be represented as a FSA with high, but finite set of states, thus does not exceed the expressiveness of FSA.

An FSA $A$ as introduced in [8] is based on a finite set of messages $\Sigma$ and generates a language $L(A)$ which enumerates the (possibly infinite) set of message sequences supported by an abstract business process. Two FSAs match, if their languages have a non-empty intersection. The intersection of two FSAs is again a FSA, which can be determined with the usual cross product construction [8]. If the resulting automaton does not contain at least one path (possibly of zero length) between a start state and an end state, its language is the empty language $\emptyset$. In this case, the business processes modeled by the FSAs are incompatible, because they do not share at least one common message sequence.

\subsection{Query Definition}

Based on these definitions, matchmaking of abstract business processes can be represented as non-empty intersection of FSA or languages being message sequence sets,

1 A net is bounded if it has a finite set of possible markings. 
that is strings. Dependent on the FSA structure, the number of strings contained in a language varies. For tree- and graph-structured (without cycles) FSA the number of message sequences is finite and can be indexed using standard techniques. However cyclic-structured FSA have an infinite number of message sequences, thus cannot be handled using standard techniques. The number of messages must be made finite first, before using standard techniques such as $\mathrm{B}^{+}$. trees. This paper focuses on cyclic structured FSA, because up to our knowledge no abstraction representing cyclic FSA as a finite set of message sequences has been presented so far.

In summary, the query consists of a FSA $A$ processed on a collection $C$ of FSA $C:=\left\{B_{1}, \ldots, B_{n}\right\}$ resulting in a subset $R$ of the collection $C$ containing all FSA $B_{i}$ having a non-empty intersection with the query FSA $A$, thus $R:=\left\{B_{i} \in C \mid L\left(B_{i}\right) \cap L(A) \neq \emptyset\right\}$.

\section{Related Work}

Existing approaches addressing this problem can be clustered into three categories. These are FSA indexing based on automata theory, graph-based and set indexing approaches. Other proposals like Access Support Relations [10], Path Index, Nested Index [6] and CH-index [13] do not suffice although from a first glance they look promising. This is because they are all based on the enumeration of objects they index, with no special provision to handle infinity of FSA languages.

\section{Indexing FSA based on automata theory}

The RE-tree [3] approach uses automata theory to build a hierarchical structure based on FSA subsumption relation to build an index. FSA subsumption defines the ordering of FSA within the hierarchical structure, unlike numeric/ lexicographic ordering as used in traditional databases. The approach allows direct indexing of FSA (including cyclicstructured FSA) and allows to perform intersection query operations. The limitation of RE-trees is that algorithms for index construction and maintanance have very high complexity. In addition, queries using the intersection operator also have a high-complexity, requiring the query FSA to be intersected with every bounding FSA along the entire height of the tree [3].

\section{Graph-based approaches}

Graph-based approaches include dataGuides [5], 1/2/Tindexes [12] and GraphGrep [17]. DataGuides and $1 / 2$ /T-indexes work by clustering together paths, such that the number of paths in the index graph is minimized, thus reducing the overall search space. In GraphGrep, graphs are indexed by computing all paths with a predefined maximum length and storing their hash values in a table, along with the graphs they reference. These approaches are inappropriate for handling cyclic struc- tured FSA, since they are all based on the enumeration of paths, without providing an appropriate way to handle cycles.

\section{Signature trees}

Signature trees [7] are used to index sets. In the case of FSA, sets could be constructed from messages of each complete path or messages of the entire FSA. A signature is constructed from the set by encoding each element of the set into a bit field of fixed length called a signature length, with a fixed number of bits of the signature length set. Bit fields are superimposed using a bitwise or operator to yield a final signature for the set. Intersection is computed using bitwise and operator. Performance analysis on signature trees have indicated that signature trees support set equality queries very well, but are poor when it comes to intersection queries [7]. Also, the data structure itself is lossy, hence introduces a lot of false matches.

\section{Approach based on N-grams}

The RE-tree approach described in the previous section addresses the stated query by relying on standard automata theory to reduce the number of required intersection calculations. We have argued that this approach is inefficient due to the high complexity of constructing the index and evaluating queries. Our approach is to apply an abstraction on the automata to derive a finite language that is indexable by standard approaches. When using this approach, it must be ensured that no false misses are introduced due to the abstraction. In particular, the abstraction $\phi$ must guarantee that if the language of FSA $A$ and $B$ match, also the abstracted representations $\phi(L(A))$ and $\phi(L(B))$ have a non-empty intersection. Formally, this can be denoted for an abstraction $\phi$ as:

$$
L(A) \cap L(B) \neq \emptyset \longrightarrow \phi(L(A)) \sqcap \phi(L(B)) \neq \emptyset
$$

The obvious approach to make the language finite is representing cycles such, that the language remains finite without introducing false misses. Consequently, abstraction introduces a loss of information due to representing several FSA as a single abstract object. In case the abstract representation $\phi(L(A))$ matches another abstract representation $\phi(L(B))$, it is assumed that all FSA associated to $\phi(L(A))$ and those associated to $\phi(L(B))$ have a non-empty intersection. This definitely is not the case, thus the abstraction introduces false matches. The abstraction proposed in this paper is based on n-grams.

In summary, the overall optimization goals of the indexing approaches based on an abstraction are: (i) minimizing the processing time (ii) guaranteeing non-occurrence of false misses and (iii) minimizing the number of false matches. The abstraction proposed in this paper is based on n-grams. 


\section{1. $n$-grams}

$\mathrm{n}$-grams have been applied in text indexing approaches in particular for substring matching [1]. The general idea behind n-grams is representing a single long string by several strings of length $n$. The length $n$ on the one hand side controls the complexity of all operations required in the concrete application scenario and on the other hand influences the fuzziness of the operation result applied on the n-gram representation. The definition of an n-gram of a word, as it is normally given in the text indexing literature [11] is defined via a function $\Phi_{n}$ as follows:

Definition 1 (n-grams)

$A$ word $\omega$ of a language is transformed to a n-gram representation by a function $\Phi_{n}$ such that: $\Phi_{n}: \Sigma^{*} \rightarrow$ list of $(\Sigma \cup\{\$\})^{n}$ and $\$ \notin \Sigma$. If $\omega=b_{1} \cdots b_{M}$ is a word with $b_{i} \in \Sigma, \Phi_{n}(\omega)=\ell_{1}, \cdots, \ell_{N}$ with $N+M+1$ such that each $\ell_{i}$ is an $n$-gram and $\ell_{i}=a_{i, 1} \cdots a_{i, n}$ for $i=1 \cdots N$

$a_{i, k}= \begin{cases}\$ & \text { if } i+k \leq n \\ \$ & \text { if } i=N \text { and } k=n \\ b_{k+i-n} & \text { otherwise }\end{cases}$

We use a special character, $\$$ which we assume not to be among the input alphabet of the automaton, to designate the start and end of a word. The special character is also used as a place holder for n-gram positions that are immediately unoccupied, e.g., a word $a$ is represented by a 3-gram as $<\$ \$ a, \$ a \$>$. Another example is the word $a b c d a b f$ being represented by the following 4-gram: $<\$ \$ \$ a, \$ \$ a b, \$ a b c, a b c d, b c d a, c d a b, d a b f, a b f \$>$. Note also that $\Phi_{n}$ is a bijective function. Due to this property, the number of words does not reduce by the transformation $\Phi_{n}$. The basic idea to make the transformed language finite is to omit duplicate occurences of a single n-gram resulting in a clustering of words in a language. The concrete definitions described next formalize the omitting of duplicate $n$-grams contained in a representation.

\section{2. $n$-gram lists}

An n-gram list is an ordered representation of an n-gram representation derived from a word, which does not contain repeating $\mathrm{n}$-grams. This is presented formally below:

Definition 2 (n-gram lists abstraction of a word) Let $p=\ell_{1}, \cdots, \ell_{N}$ be an $n$-gram representation of a word accepted by an automaton. $p$ is transformed by an abstraction function $\varphi$ to $p^{\prime}$, i.e., $\varphi(p)=p^{\prime}$, where $\varphi\left(\ell_{1}, \cdots, \ell_{N}\right)=<\ell_{1}^{\prime}, \cdots, \ell_{N^{\prime}}^{\prime}>, N^{\prime} \leq N$ $\ell_{k}^{\prime}= \begin{cases}\ell_{k} & \text { if } \nexists j<k . \ell_{j}=\ell_{k} \\ \epsilon & \text { otherwise }\end{cases}$

The $\epsilon$ denotes the empty list, that is adding the element to the list keeps the list unchanged. Due to the fact, that the resulting n-gram list does not contain duplicates the number of elements contained in the list is fixed, opposed to the number of characters contained in a word of the language. Here the representation is made finite by clustering several words having the same n-gram list representation. This definition can easily be adapted to languages by applying the $\varphi$ transformation on each n-gram representation of the words contained in the language.

Definition 3 (n-gram lists abstraction of language)

Let $A$ be an FSA and $L(A)=\left\{\omega_{1}, \cdots, \omega_{N}\right\}$ the corresponding possibly infinite language. The n-gram list abstraction $\phi_{n}$ is defined as: $\phi_{n}\left(\omega_{i}\right):=\varphi\left(\Phi_{n}\left(\omega_{i}\right)\right)$ and $\phi_{n}(L(A)):=\bigcup_{i=1}^{N} \varphi\left(\Phi_{n}\left(\omega_{i}\right)\right)$

The definitions are illustrated on behalf of the introductory customer process depicted in Figure 1a). The language constructed by this FSA can be specified as $\{$ ird,iraoac, iraodoac,...,iraodo...doac $\}$, where the first character of the message names are used. Applying the n-gram list abstraction results in the following:

$$
\begin{aligned}
& \text { - } \phi_{2}(\text { ird }):=<\$ i, i r, r d, d \$> \\
& \text { - } \phi_{2}(\text { iraoac }):=<\$ i, i r, r a, a o, o a, a c, c \$> \\
& \text { - } \phi_{2}(\text { iraodoac }):=<\$ i, i r, r a, a o, o d, d o, o a, a c, c \$> \\
& \text { - } \phi_{2}(\text { iraododoac }):=<\$ i, i r, r a, a o, o d, d o, o a, a c, c \$>
\end{aligned}
$$

In the last example, the repeating 2-grams od and $d o$ are only contained once in the list. Obviously, the repeated execution of the loop does not introduce new 2-grams, thus does not change the 2-gram list representation. As a consequence the above listed three 2-gram lists are the finite abstraction of the FSA depicted in Figure 1a) used for indexing. In particular each 2-gram list is stored as a string in a classical database.

Figure 2a) depicts the subset of the language captured by the 2-gram list $<\$ i, i r, r a, a o, o d, d o, o a, a c, c \$>$. Unfortunately, the 2-grams $r a$ and $o a$ have a common last character, thus the preceding 2-gram might be either $a c$ or $a o$. Based on this observation, the word iraodoaoac is also accepted by the same 2-gram list being one example of a false match introduced by this abstraction. The automaton representing the set of words clustered by the above 2-gram list is depicted in Figure 2b).

The cluster size, which corresponds to the number of potential false matches, depends on the size of $n$. Using a 3gram representation of the above example FSA depicted in Figure 2a) results in the following finite 3-gram list representation:

- $\phi_{3}(i r d):=<\$ \$ i, \$ i r, i r d, r d \$>$

- $\phi_{3}($ iraoac $):=<\$ \$$, \$ir, ira, rao, aoa, oac, ac\$>

- $\phi_{3}($ iraodoac $):=<\$$ i, \$ir, ira, rao, aod, odo, doa, oac, $a c \$>$ 


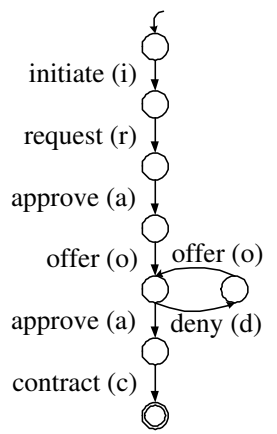

(a)

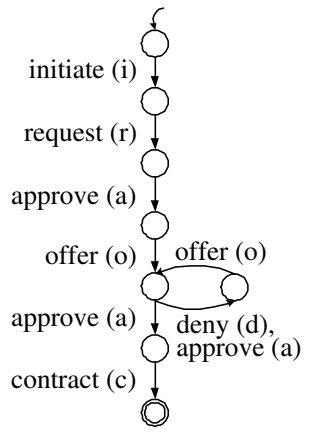

(b)
Figure 2. (a) Original Process. (b) Derived Process Class

- $\phi_{3}($ iraododoac $):=<\$ \$ i, \$ i r$, ira, rao, aod, odo, dod, doa, oac, ac $\$>$

- $\phi_{3}($ iraodododoac $):=<\$ \$ i, \$ i r$, ira, rao, aod, odo, dod, doa, oac, ac\$>

In this example, the size of the 3-gram list representation has increased. As a consequence of the additional information the cluster represented by

$<\$ \$ i, \$ i r$, ira, rao, aod, odo, dod, doa, oac, ac $\$>$ has decreased significantly, because the variation of $a c$ and $a o$ observed above is no longer possible due to the additional character contained in a single 3-gram. The related cluster to the 3-gram list represents exactly the requested set of strings, thus no false matches are introduced.

The $\mathrm{n}$-gram list transformation can be used for indexing FSA by calculating the finite set of n-gram lists by omitting repeating $\mathrm{n}$-grams per $\mathrm{n}$-gram list and relating them to the FSA itself. The query can be performed by transforming the query FSA in accordance to the transformation function used for initiating the index and check for equivalent n-gram lists within the index by a string comparison in the database. The query evaluation can formally be described as:

\section{Definition 4 Query evaluation of n-gram lists}

Let $C:=\left\{B_{1}, \cdots, B_{N}\right\}$ be the set of FSA in the collection $C$ and $A$ the query automaton. The query result $R$ contains FSA $B_{i}$ having at least one $n$-gram list in common $R:=\bigcup_{p \in \phi_{n}(L(A))} \bigcup_{i=1}^{N} \Theta_{n}\left(p, B_{i}\right)$ with $\Theta_{n}\left(p, B_{i}\right):= \begin{cases}\left\{B_{i}\right\} & \text { if } p \in \phi_{n}\left(L\left(B_{i}\right)\right) \\ \emptyset & \text { otherwise }\end{cases}$

It can be shown that the n-gram list abstraction does not introduce false misses.

\section{Implementation}

The matchmaking and n-gram indexing approach described in this paper is applied to service discovery within the web services infrastructure [21], where service consumers want to search for relevant service providers. The de facto standard for service discovery within the web service infrastructure is UDDI. UDDI provides a limited querying functionality based on flat key comparison e.g., business or service keys, without having the ability to deal with multiple dimensions of a service e.g., semantic, quality of service and workflow aspects. The approach described in this paper is complementary to UDDI in that it adds the ability to find relevant services, taking into account not only the name and category of the service, but also workflow information. The services are assumed to be using the same data dictionary provided by organizations like RosettaNet for message names, thus no semantic reasoning is required as we already pointed out at the beginning of this paper.

\subsection{Framework}

An infrastructure realizing the two query dimensions of a service has two major components, namely the UDDI component to support standard UDDI queries and a process engine component, to support the matching of workflows based on the approach described in this paper. Thus the query input contains the UDDI part taking UDDI specific parameters and the workflow part described using standard workflow standards such as BPEL. The two query parts are passed to the respective engines i.e., the UDDI part of the query is parsed to the UDDI engine such as jUDDI [9] and the workflow (BPEL) part is passed to another component that converts BPEL to FSA. We have implemented such a BPEL to FSA convertor and it is published in a separate paper [20]. After the conversion, the FSA is passed to the process engine for computing the workflow matching using the approach described in this paper.

\subsection{Indexing}

We implemented an API for transforming the language accepted by an FSA into a finite set of $\mathrm{n}$-gram lists. This is achieved by removing cycles by observing that the number of n-grams in every FSA is finite; thus by removing repeating n-grams along a path, we can represent the language of any FSA finitely, using the $\mathrm{n}$-gram representation of its language where duplicates are omitted. Thus the resulting $n$ gram lists, which are words abstracted from FSA words, are mapped to relational tables where they are used for indexing based on standard B+-tree data structures. The n-gram lists contain references to FSA they are computed from. 


\subsection{Preliminary Evaluation}

We carried out an initial evaluation of the approach described in this paper. The goal was to find the performance factor achieved by using the indexing approach against sequential searching. We were also interested in finding out what influences the complexity of the indexing approach from an empirical view point.

System Parameters The experiments were conducted on a Dell machine, with a Pentium 4 processor $2.00 \mathrm{GHz}$ clock speed and 512 MB RAM with total disk space of 74 GB. The machine was lightly loaded and running under Windows XP operating system. Persistent storage and data management was based on MySQL server 4.0, also running on the same machine. JBOSS 3.2.5 application server provided the J2EE environment for the matchmaking engine and container managed persistency (CMP) was the used persistent manager. No buffering/ caching was allowed and all tests were run under cold start conditions. Each query was averaged over several runs and only the averaged values were considered in the final result set.

Data set Specification The used data set is business process data based on the RosettaNet PIP specification. RosettaNet PIPs are atomic processes that can be combined to form larger sets of business processes. The data set com-

\begin{tabular}{|r|r|r|r|}
\hline \hline$N$ & states & $|\Sigma|$ & transitions \\
\hline \hline 274 & 6733 & 3747 & 6557 \\
\hline 548 & 13466 & 7494 & 13114 \\
\hline 822 & 20114 & 11222 & 19642 \\
\hline
\end{tabular}

Table 1. FSA complexity

prised a total of 822 business processes which were represented as FSA. The FSA were both cyclic and non-cyclic structured. The cyclic FSA were simple cycles only, with no complex cycles (nested cycles which are joining at least one state). The FSA were grouped into three sets of different sizes of 274,548 and 822 . This allows to test perfor-

\begin{tabular}{|r|r|r|r|r|}
\hline \hline$N \backslash n$ & 1 & 2 & 3 & 4 \\
\hline \hline 274 & 3228 & 3665 & 3665 & 4210 \\
\hline 548 & 6456 & 7330 & 7330 & 8240 \\
\hline 822 & 9760 & 11276 & 11276 & 20114 \\
\hline
\end{tabular}

Table 2. number of $n$-gram lists per data set/ look-back matrix

mance while varying the size variable, thus allowing to test the influence of data set size on performance. The n-gram length was also varied from 1 to 4 , for each data set; this allows to measure the influence of n-gram length on performance as well as determine false match rate with respect to the n-gram length. The number of state, message, transition and n-gram list objects stored in the database were recorded for each data set and n-gram length. This information gives an idea of the complexity of the data set. Tables 1 and 2 summarize this information.

Results During the preliminary evaluation, processing time of queries quantified in milliseconds was measured. The best, average and worst case performances on the 548 data set were $33 \mathrm{~ms}, 177 \mathrm{~ms}$ and $1437 \mathrm{~ms}$ respectively when using index search and about $17 \mathrm{~s}, 29 \mathrm{~s}$ and $46 \mathrm{~s}$ with sequential search. More detailed measurements were also taken for different n-gram lengths and data sets.

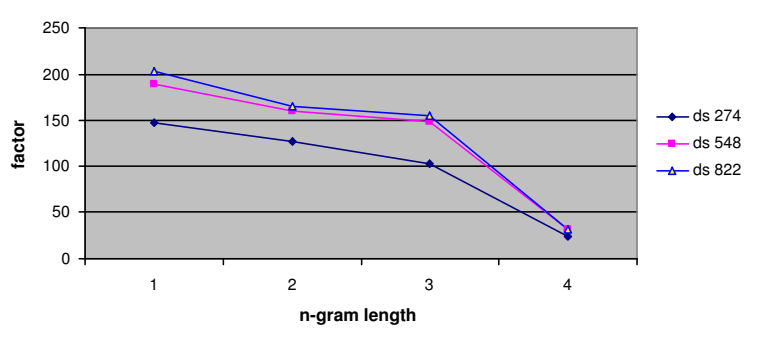

Figure 3. Performance Factor

Figure 3 depicts the search improvement factors of our indexing approach against sequential scanning for different n-gram lengths and data sets. The figure shows that for the smallest data set of 274 FSA, index search was faster than sequential search by a factor of 147 for n-gram length of 1 declining to a factor of 24 for n-gram length of 4 . A similar trend can be observed for the data set of size 548 and 822. This behavior can be easily explained.

Increasing the used n-gram length also increases the number of $n$-gram lists to be maintained in the database. Thus, the best search performance is achieved when n-gram length is 1 , because the number of n-gram sequences is lowest. This is because the number of comparisons to be made is lowest as shown in Table 2. In addition, the length of $n$ gram lists becomes longer as the $n$-gram length is increased since more messages are considered when constructing $n$ grams. The result is that both search and storage complexities increase when n-gram length increases. The benefit of increasing the n-gram length is that it increases the quality of search results i.e., the number of false matches decreases. There is always a trade-off between attaining a high quality of search results and attaining higher levels of performance. 
As shown in Figure 3 the best performance gain of 203 times is achieved when n-gram length is 1 . For the reasons explained above, the number of false matches is also highest at this point.

Another feature of our index to be observed from Figure 3 is that performance gain over sequential scanning increases with increase in data set size. For example, for all n-gram lengths from 1 to 4, performance gains are highest for the largest data set as shown in the figure. Thus our indexing approach gets better as the data set size increases.

Storage complexity The amount of storage required by the index is significantly more than that required without using the index and it increases as the n-gram length increases. However efficient text compression techniques e.g., described in [18] can be used to compress individual ngrams thus saving on storage space. n-gram lists from the query FSA must be compressed using the same technique before querying the database.

\section{Summary and Future Work}

The paper presented an approach for efficiently finding service providers with matching business processes. It proposed modeling business processes using FSA. The semantics for comparing business processes for a match is nonemptiness of FSA intersection. The approach is based on the creation of an index on the set of potentially infinite message sequences supported by each provider, and using this for efficient searching. Traditional databases cannot handle infinite message sequences and they also do not support intersection operations. We reduce the infinite language of an FSA to a finite one by using non-repeating n-grams to represent words of the language. We replace the intersection operator with the equality operator on n-gram lists, which are finite. We have implemented and evaluated the approach with RosettaNet data and initial results are very encouraging: there is a definite net improvement over sequential scanning. Future work is carrying-out a more detailed evaluation of the approach along with full complexity analysis and investigation of highly complex FSA.

\section{References}

[1] R. A. Baeza-Yates. Text retrieval: theory and practice. In J. van Leeuwen, editor, Proceedings of the 12th IFIP World Computer Congress, pages 465-476, Madrid, Spain, 1992. North-Holland.

[2] T. Berbers-Lee, J. Hendler, and O. Lassila. The semantic web. Scientific America, 284(5):34-43, 2001.

[3] C. Chan, M. Garofalakis, and R. Rastogi. Re-tree: an efficient index structure for regular expressions. The $V L D B$ Journal, 12(2):102-119, 2003.
[4] J. Esparza and M. Nielsen. Decibility issues for Petri nets a survey. Journal of Informatik Processing and Cybernetics, 30(3):143-160, 1994.

[5] R. Goldman and J. Widom. Dataguides: Enabling query formulation and optimization in semistructured databases. In Proceedings of the 23rd VLDB Conference, Athens, Greece, 1997.

[6] E. Gudes. A uniform indexing scheme for object-oriented databases. Information Systems, 22(4):199-221, 1997.

[7] S. Helmer and G. Moerkotte. A performance study of four index structures for set-valued attributes of low cardinality. The VLDB Journal, 12(3):244-261, 2003.

[8] J. E. Hopcroft, R. Motwani, and J. D. Ullman. Introduction to Automata Theory, Languages, and Computation. Addison Wesley, 2001.

[9] juddi. juddi. http://ws.apache.org/juddi.

[10] A. Kemper and G. Moerkotte. Access support relations: An indexing method for object bases. Information Systems, 17(2):117-145, 1992.

[11] J. Y. Kim and J. Shawe-Taylor. Fast string matching using an $n$-gram algorithm. Software - Practice and Experience, 24(1):79-88, 1994.

[12] T. Milo and D. Suciu. Index structures for path expressions. Lecture Notes in Computer Science, 1540:277-295, 1999.

[13] T. A. Mueck and M. L. Polaschek. A configurable type hierarchy index for oodb. The VLDB Journal The International Journal on Very Large Data Bases, 6(4):312-332, 1997.

[14] M. Paolucci, T. Kawmura, T. Payne, and K. Sycara. Semantic matching of web services capabilities. In Proceedings of the First International Semantic Web Conference on The Semantic Web, pages 333-347. Springer-Verlag, 2002.

[15] J. L. Peterson. Petri Net Theory and the Modeling of Systems. Prentice-Hall, 1981.

[16] A. ShaiklAli, O. F. Rana, R. Al-Ali, and D. W. Walker. Uddie: An extended registry for web services. In Proceedings of the 2003 Symposium on Applications and the Internet Workshops (SAINT-w03). IEEE Computer Society, 2003.

[17] D. Shasha, J. T.-L. Wang, and R. Giugno. Algorithmics and applications of tree and graph searching. In L. Popa, editor, Symposium on Principles of Database Systems, pages 39-52. ACM, 2002.

[18] A. B. Tucker, editor. Computer science handbook. Chapman \& Hall /CRC, second edition, 2004.

[19] A. Wombacher, P. Fankhauser, B. Mahleko, and E. Neuhold. Matchmaking for business processes based on choreographies. International Journal of Web Services, 1(4):14-32, 2004.

[20] A. Wombacher, P. Fankhauser, and E. Neuhold. Transforming bpel into annotated deterministic finite state automata for service discovery. In IEEE International Conference on Web Services (ICWS 2004), pages 316-323, Los Alamitos, California, 2004. IEEE Computer society.

[21] A. Wombacher, B. Mahleko, and E. Neuhold. Ipsi-pf: A business process matchmaking engine. In IEEE Conference on E-Commerce Technology (CEC 2004), pages 137-145, Los Alamitos, California, 2004. IEEE Computer society. 